\title{
HIGH TEMPERATURE AND HIGH RESOLUTION UV PHOTOELECTRON SPECTROSCOPY USING SUPERSONIC MOLECULAR BEAMS
}

\author{
LAI-SHENG WANG ${ }^{1}$, J.E. REUTT-ROBEY ${ }^{2}$, B. NIU ${ }^{1}$, Y.T. LEE ${ }^{1}$ AND D.A. SHIRLEY ${ }^{1}$ \\ ${ }^{1}$ Materials and Chemical Sciences Division, Lawrence Berkeley Laboratory, 1 Cyclotron Road and \\ Department of Chemistry, University of Califomia, Berkeley, CA 94720 (U.S.A.) \\ ${ }^{2}$ Deparment of Chemistry, University of Maryland, College Park, MD 20742 (U.S.A.)
}

\section{ABSTRACT}

A high temperature molecular beam source with electron bombardment heating has been bu.lt for high resolution photoelectron spectroscopic studies of high temperature species and clusters. This source has the advantages of: (1) producing an intense, continuous, seeded molecular beam, (2) eliminating the interference of the heating mechanism from the photoelectron measurement. Coupling the source with our hemispherical electron energy analyzer, we can obtain very high resolution HeI $\alpha$ ( $584 \AA$ ) photoelectron spectra of high temperature species. Vibrationally-resolved photoelectron spectra of PbSe, As2, As4, and $\mathrm{ZnCl}_{2}$ are shown to demonstrate the performance of the new source.

\section{INTRODUCTION}

High temperature photoelectron spectroscopy (PES) offers opportunities to study transient species, and vapor species and clusters of solid substances (1). It has been an active field of research and continues to provide important experimental results concerning the electronic properties of radical species, vapor molecules and clusters in the gas phase $(2,3)$. However, high temperature PES has been hindered by two factors, namely, the difficulty of generating high temperature species and the effect of the high temperature oven on the photoelectron measurement. Consequently, high resolution PE spectra of high temperature species are still scarce. In this paper, we describe a new high temperature molecular beam source which allows us to do very high resolution PES of high temperature species.

Our photoelectron spectrometer, which is composed of a rare gas discharge lamp, a quadrupole mass spectrometer and a hemispherical energy analyzer with a multichannel detector, has been described in detail before (4). It has a resolution of about $11 \mathrm{meV}$. High resolution PE spectra of many gas phase molecules have been obtained with this spectrometer (5). Interested readers are referred to Ref.(4) for the details of the spectrometer. In this paper, our attention will be focused on the details of the high temperature molecular beam source, its design, construction and performance. Coupling this source with the photoelectron spectrometer, we have achieved a resolution in the range of $13-16 \mathrm{meV}$ for high temperature PES studies. 


\section{EXPERIMENTAL}

\subsection{Design Considerations}

Our design criteria were dictated by the photoelectron spectrometer. A high number density $\left(\sim 10^{12}-10^{13}\right.$ molecules $\left./ \mathrm{cm}^{3}\right)$ is necessary for the photoelectron measurement. This number density, further, must be produced without a significant magnetic field, which would perturb the photoelectron measurement. In order to obtain well-resolved PE spectra, internal cooling of the high temperature species is essential. This poses a conflicting requirement with the high temperature environment necessary. Fortunately, this can be resolved through the seeded supersonic molecular beam technique. In brief, what we need is a source that can cleanly generate an intense, continuous and internally-cooled molecular beam.

A number of designs were considered for this source. The very successful laser evaporation technique was attractive, in that it is simple to construct and can evaporate virtually any materials (6). The low average number density output of this technique, however, precludes its use in our photoemission studies. A direct resistive heating source has the advantage of simplicity and ease to operate (7). But the enormous current necessary in this method would not be desirable. A pulsed RF heating source (8) has been successfully applied to UV photoemission studies. The disadvantage is that the photoelectron measurement must be gated with respect to the heating periods, reducing the collecting efficiency of the electron spectrometer.

An electron bombardment heating source provides an attractive alternative (9). It requires relatively low heating current ( $<40 \mathrm{~A}$ for a $0.51 \mathrm{~mm}$ tungsten filament). Further, the filament can be wound noninductively and thus introduce little magnetic field. The heating power will be controlled by the thermionic emission current and the accelerating high voltage. So continuous high power can be achieved easily. Therefore, the electron bombardment heating technique became the choice in our design.

\subsection{Construction}

The first thing to consider in building an e-beam heating source is the ability to apply high enough accelerating voltage to achieve the desirable heating power without having electrical breakdown at the same time. In our case, we would like to have up to $1 \mathrm{~A}$ emission current and up to $2.5 \mathrm{KV}$ accelerating voltage. Since the vacuum in our molecular beam source chamber with a molecular beam running is $\sim 10^{-4}$ torr, all the floated components must be placed in a differentially-pumped vacuum region and operated at a vacuum better than $\sim 10^{-5}$ torr.

A schematic cross-sectional view of the e-beam heating oven is shown in Fig. 1. It consists primarily of a $0.51 \mathrm{~mm}$ coiled W filament (15, Fig.1) for thermionic emission, a Mo cylinder electrostatic deflector (9, Fig. 1), inner and outer $W$ vacuum jackets (12 and 13, Fig. 1), a graphite crucible (11, Fig. 1) and a carrier 


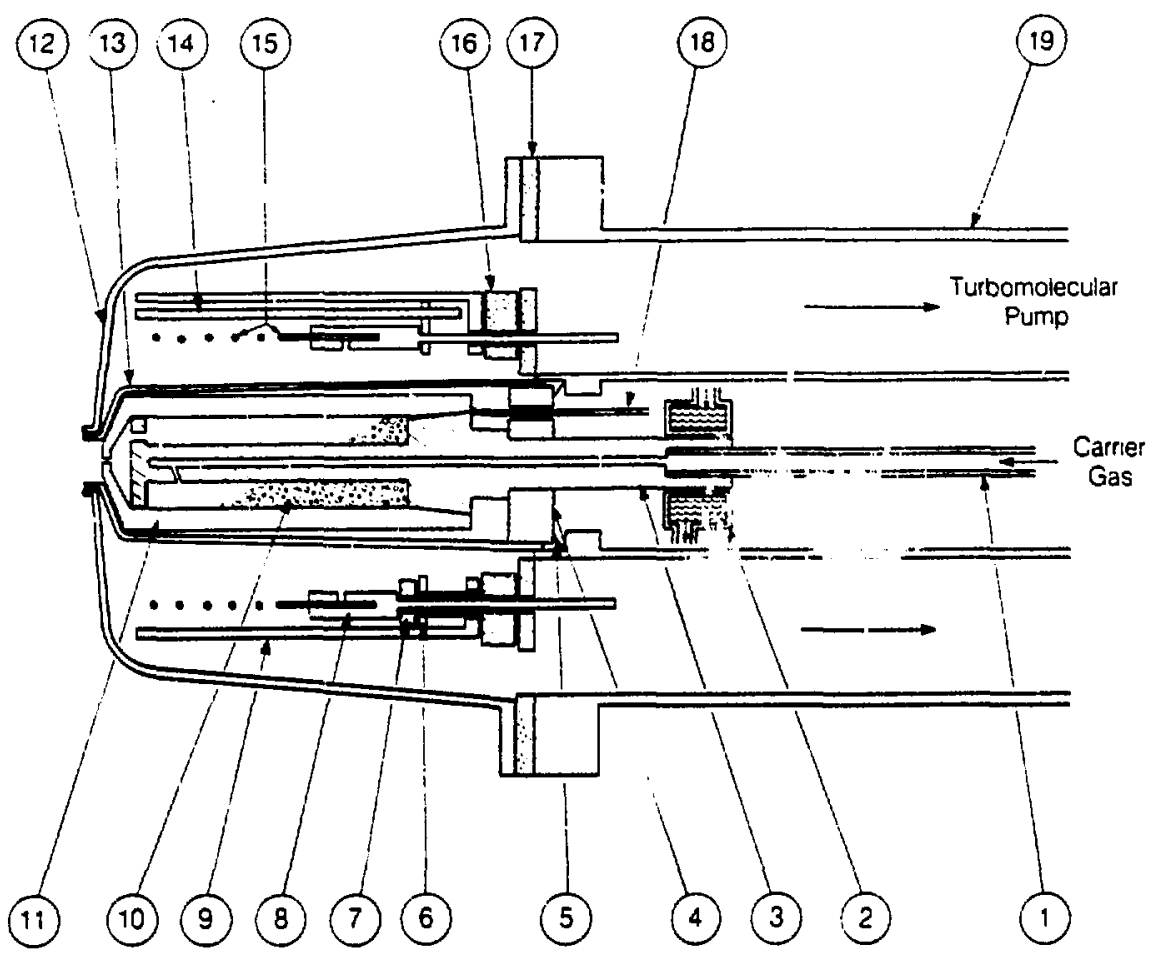

Fig. 1. A cross sectional view of the oven assembly. (1) Ta carrier gas inlet tube, (2) water cooling block, (3) graphite crucible cap, (4) graphite radiation shield, (5) Mo springy conduction barrier, (6) Mo retainer, (7) ceramic insulator, (8) Mo filament mount post, (9) Mo electrostatic deflector and radiation shield, (10) sample, (11) graphite crucible body, (12) outer W (or Ta) vacuum jecket, (13) inner W (or Ta) vacuum jacket, (14) ceramic filament support rod, (1 W filamert, (16) ceramic high voltage standoff, (17) Mo (or ceramic) support plate for filament posts, (18) thermocouple, (19) stainless steel triple-tubed flange section (shown in two-fold symmetry for easier viewing). Not shown are the bus lines, some radiation shields, and $W$ wires for supporting the filament.

gas inlet system (1, Fig. 1). The $W$ filament is noninductively wound, supported by a ceramic filament support (14, Fig. 1) and screw-mounted onto two Mo filament mount posts (8, Fig. 1). The bus (see 17, Fig. 3), supplying power for the filament and running through a triple-tubing section (19, Fig. 1), is not shown in Fig. 1. The filament and the electrostatic deflector are floated at negative high voltage with respect to the surroundings, and are insulated from the surroundings through the ceramic high voltage standoff (16, Fig. 1). Hence, the electrons emitted from the filament are accelerated toward the inner vacuum jacket, which in turn heats up the crucible radiatively.

The inner and outer vacuum jackets define a separate vacuum region which is differentially pumped by a $200 \mathrm{l} / \mathrm{s}$ Turbo pump (see Fig. 3). This is necessary to operate under high voltage condition. A pair of bevelled Mo spacers (5, Fig. 1) are placed at the end of the inner $W$ jacket. Being springy, these spacers serve as a 


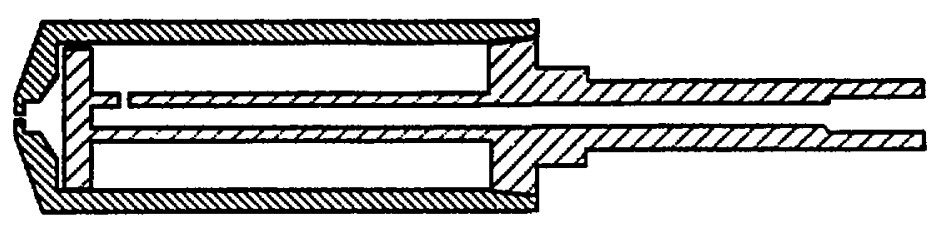

(a)

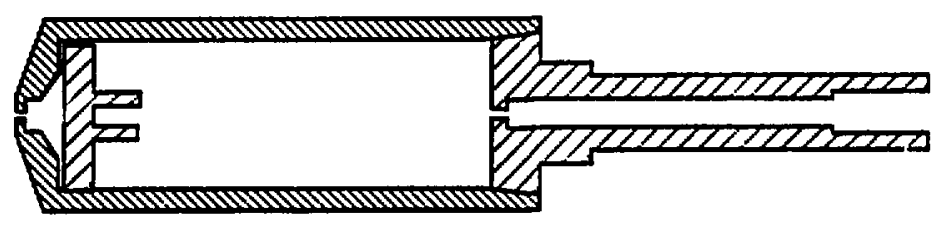

(b)

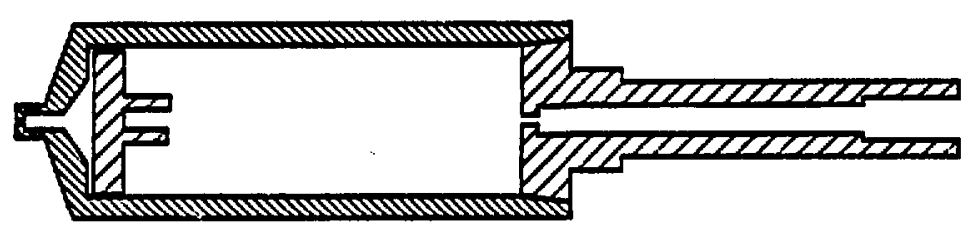

(c)

$-2.54 \mathrm{~cm}=$

Fig. 2. The three graphite crucible configurations.

differential pumping barrier between the filament region and the main beam source chamber during a whole heating cycle.

The crucible is spring-pressed against the inner jacket to allow thermal expansion during heating ( 3 and 4, Fig. 3). The top of the crucible makes direct contact with the inner jacket, and therefore is the hottest part of the crucible. This is essential to assure the nozzle is not clogged. A $4.76 \mathrm{~mm}$ (3/16 in.) o.d. Mo tube (1, Fig. 1) is tightly fitted into the end of the graphite crucible cap (also see Fig. 2). Graphite cement is applied at this joint for sealing. Since the thermal expansion coefficient of graphite is the smallest among the common high temperature materials, it is essential to cool the Mo/graphite joint (2, Fig. 1).

The design and fabrication of the $W$ filament, the vacuum jackets and the graphite crucible deserve special mention. The $W$ filament is customer-designed and can be purchased from Emissive Products (part \# 060183). The W vacuum jackets were fabricated by a chemical vapor deposition process. Tantalum was found to be applicable as a vacuum jacket material. The Ta vacuum jackets can be easily cold-formed out of $1 \mathrm{~mm}$ Ta sheets.

A central question in making the crucible was the ability to load samples and to seal off the crucible. High density graphite was chosen as the crucible material, because of its chemical inertness and its ease to be machined. Three kinds of 


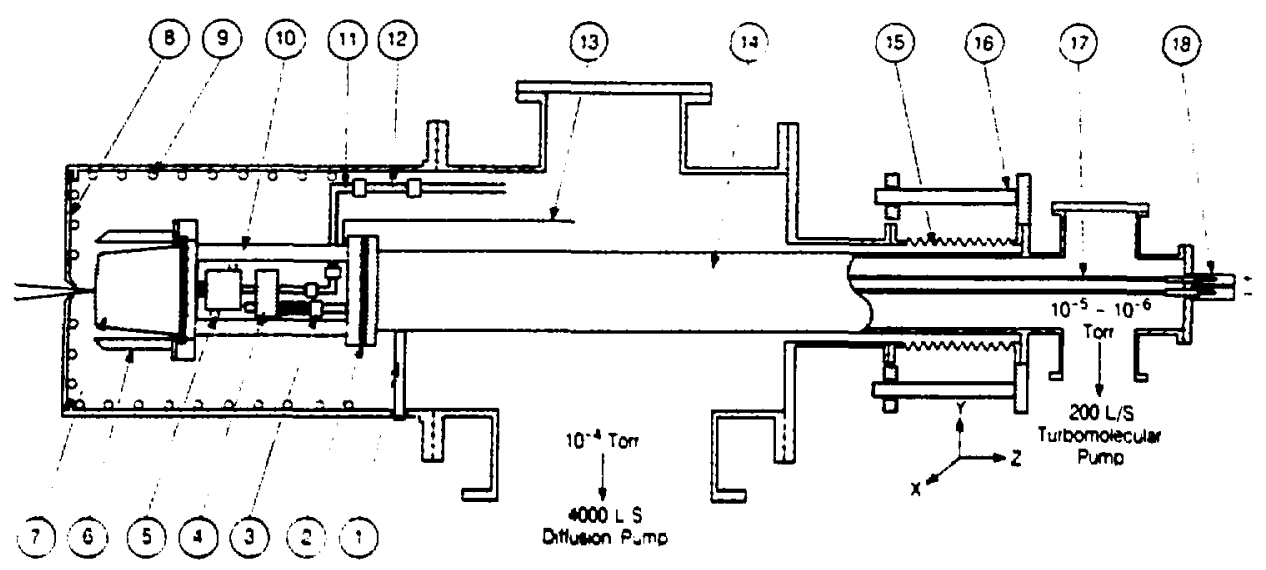

Fig. 3. A schematic view of the high temperature molecular beam source. (1) rods for fine alignment of the nozzle and skimmer (shown one of three), (2) ceramic spacer, (3) guiding rod, spling, and lock nuts for spring loading (shown one of three), (4) clamp base plate (receiving spring force), (5) water cooling block, (6) water cooled thermal shield, (7) oven assembly (shown in Fig. 1), (8) reducer cap, (9) water cooling coil, (10) triple-tubed flange, (11) carrier gas inlet line, (12) ceramic insulating tube, (13) emission return conductor, (14) stainless steel arm, (15) bellow, (16) $X Y Z$ translation stage, (17) filament busbar, (18) vacuum feedthru. Not shown are the various water lines and thermocouples. All water lines have electrical breaks and are made of flexible tubing or plastic tubing to free the motion of the stainless steel arm.

crucible configurations were used, as shown in Fig. 2. They are composed of three parts, the crucible body with a nozzle, the crucible cap with the gas inlet, and the front disk to prevent sample from getting to the nozzle. It is essential to polish the crucible body and the crucible cap contact surfaces and have a tight fit in order to prevent leaks from this joint, which is further applied with graphite cement from outside. In configuration (a), the gas inlet opening is placed at front to prevent it from getting clogged. The clogging was not a serious problem and the configuration (a) can be simplified to (b), with which a sample cell can be used if desired. It should be kept in mind that there is a temperature gradient along the crucible body with the nozzle being at the hottest part, which posed a conflicting condition for the desire to generate internally-cooled beams. Configuration (c) was designed as a compromise, for the nozzle temperature gets reduced to some degree compared with (a) and (b) as a result of more radiation loss. The graphite cement applied on the crucible can be chipped off after an experiment and the crucible can be reused. The nozzle sizes used range from $0.1 \mathrm{~mm}$ to $0.2 \mathrm{~mm}$ i.d.

The overall view of the beam source is shown in Fig. 3. A conical skimmer of $5.1 \mathrm{~mm}$ diameter is used and is not heated. The skimmer diameter is much bigger than normal to prevent clogging of the skimmer during the course of an experiment. The nozzle/skimmer alignment is achieved through the XYZ 


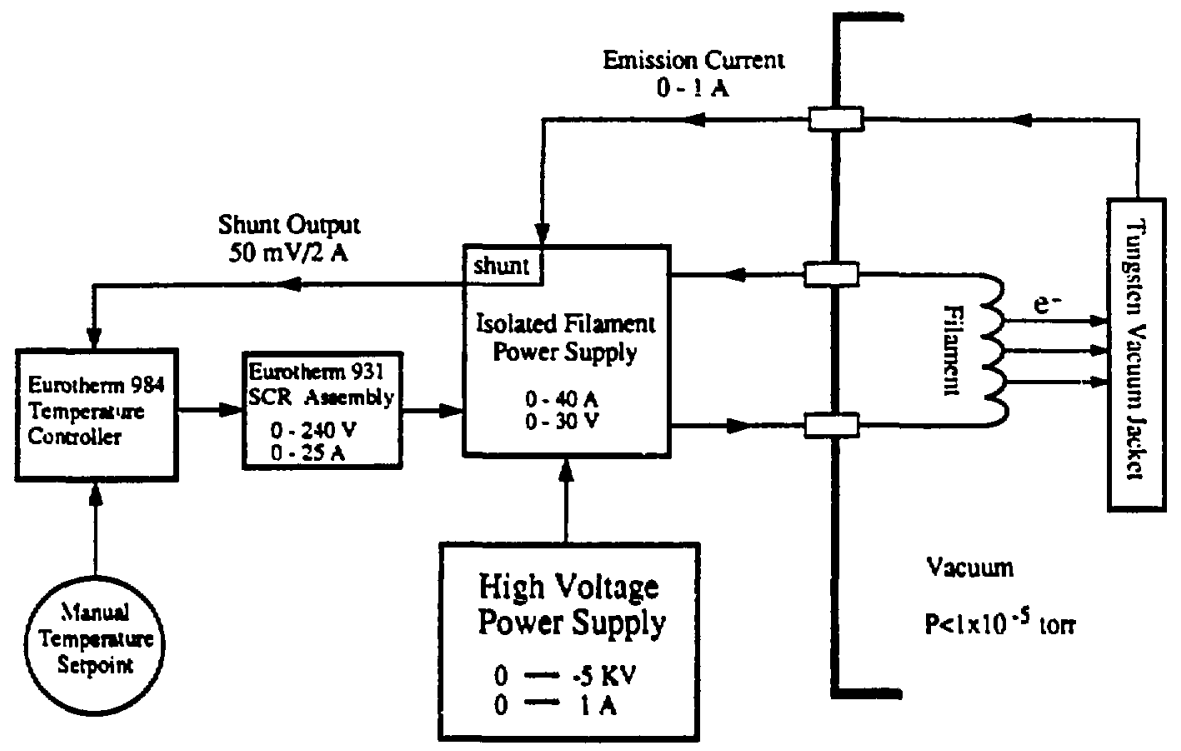

Fig. 4. Schematic diagram of the electron bombardment heating system.

translation stage (15 and 16, Fig. 3 ) and the fine alignment rods (1, Fig. 3). The outer vacuum jacket is shielded with a stainless steel water cooling cylinder $(6$, Fig. 3). Not shown is the front shielding which consists of five layers of $\mathrm{Ta}$ sheet. A ceramic spacer ( 2 , Fig. 3 ) electrically insulates the oven system from ground. A copper wire is connected to this part to collect the emission current, which is used as feedback for the filament heating control system (see below). All the cooling water lines and gas inlet line (11, Fig. 3 ) are also electrically isolated from the oven system.

The $200 \mathrm{l} / \mathrm{s}$ Turbo pump evacuates the filament region through a long $10 \mathrm{~cm}$ i.d. stainless steel tubing (14, Fig. 3). The filament bus bars (17, Fig. 3), running through this tubing, are connected with the Mo filament mount posts (8, Fig. 1). The crucible gas inlet tubing is clamped to a clamp base plate $(4$, Fig. 3$)$ and spring-loaded (3, Fig. 3). The crucible receives the spring force. The main beam source chamber is pumped by a $4000 \mathrm{l} / \mathrm{s}$ diffusion pump with a water trap.

\subsection{Operation}

The oven system is accessed from the front. The crucible is first sealed off with the desired sample. Sample changing is done simply by taking off the vacuum jackets and changing the crucible.

A schematic diagram of the e-beam heating system is shown in Fig. 4. The temperature of the oven is controlled by the emission current and the applied accelerating voltage. The desired emission current is manually set on a proportional bandwidth controller (Eurotherm 984), which provides a voltage 
pulse to trigger a Silicon Controlled Rectifier assembly ( Eurotherm 931, SCR assembly). The SCR assembly then outputs phase-controlled power (up to $240 \mathrm{~V}$, 25 A peak power) to the primary of the transformer in the isolated filament power supply. The output direct current from the filament supply (up to $40 \mathrm{~A}, 30 \mathrm{~V}$ ) is floated at a negative high voltage manually set on the high voltage supply (up to -5 $\mathrm{KV}, 1 \mathrm{~A}$ ) and is sent through the $\mathrm{W}$ filament. The emission current is collected and fed back to the temperature controller. The controller reaches a stable condition once the amount of the emission current equals the set value.

The oven temperature is functionally dependent on the emission current and the high voltage, which can be changed independently to arrive at a different temperature. The temperature is measured at the back of the crucible (18, Fig. 1) with Type $\mathrm{C}(\mathrm{W} / \mathrm{Re})$ or Type $\mathrm{K}(\mathrm{Cr} / \mathrm{Al})$ thermocouples. Due to the thermal gradient along the crucible and the thermal loss at the back of the crucible, the measured temperature is normally much lower than the real oven temperature.

During an experiment, the temperature is slowly increased to let the oven system outgas with carrier gas running. The temperature is continuously increased until there is an appreciable evaporation. This is monitored by a quadrupole mass spectrometer. The temperature, which is only relative, is then stabilized at a value where a satisfactory photoelectron count is read. The whole spectrum will be taken at this temperature. About 0.1-0.2 mole of material can be charged into the crucible. This would usually last for more than $\mathbf{2 0}$ hours, which is enough for one experiment.

The power supply system can output up to $5 \mathrm{KW}$ heating powar $(1 \mathrm{~A} \times 5 \mathrm{KV})$. The actual power output is limited by the breakdown voltage, which depends upon the temperature and the vacuum. We were able to operate the oven source at $\sim 2000 \mathrm{~K}$ with $1 \mathrm{KW}$ heating power. The thermal inhomogeneity along the crucible often makes it difficult to evaporate refractory materials. More efficient pumping of the filament region to suppress high voltage breakdown and more extensive thermal shielding at the back of the crucible can make the source work at even higher temperature.

\section{RESULTS AND DISCUSSION}

With this source, we have obtained high resolution photoelectron spectra from three kinds of molecules, namely, the heavy Group IV-VI diatomics, the clusters of the Group $V$ elements, and the first row transition metal dihalides. To show the performance of the source, we will discuss four molecules among these: $\mathrm{PbSe}, \mathrm{As}_{2}, \mathrm{As}_{4}$ and $\mathrm{ZnCl}_{2}$.

\section{$3.1 \mathrm{PbSe}$}

The vibrationally-resolved photoelectron spectrum of $\mathrm{PbSe}$ is shown in Fig. 5. This spectrum was taken with a crucible of configuration (a) in Fig. $2(0.13 \mathrm{~mm}$ 


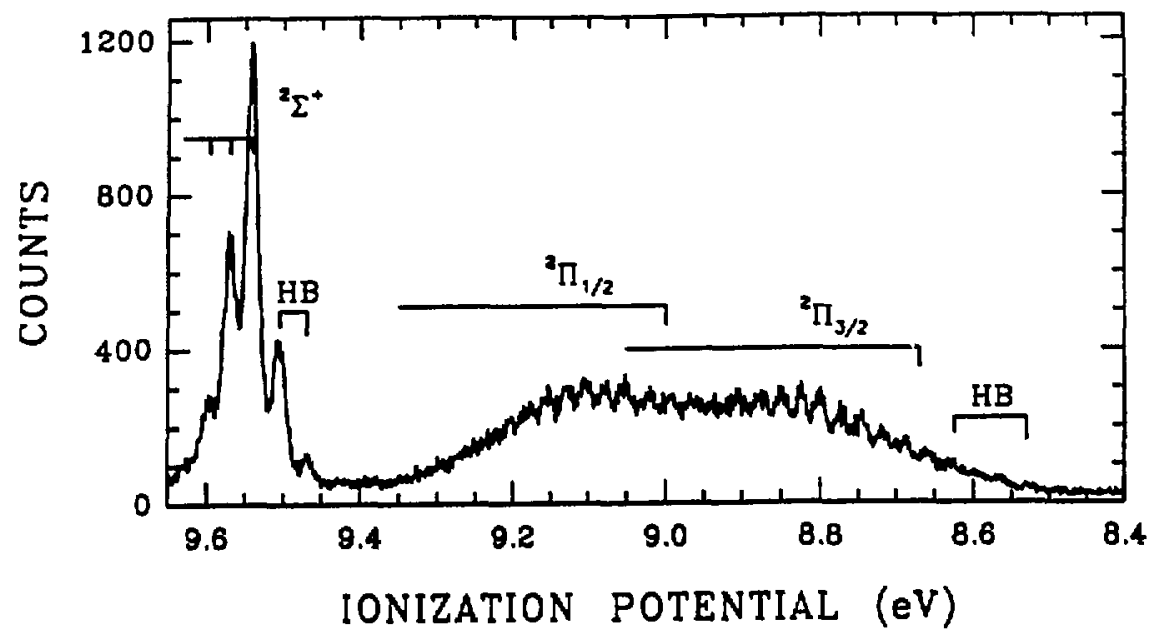

Fig. 5. The Hel Photoelectron spectrum of PbSe. HB stands for the hot band transitions.

dia. nozzle size). The carrier gas was 500 torr Ne and the oven temperature was held at about $1150 \mathrm{~K}$. The supersonic expansion was not particularly strong in this crucible configuration, giving appreciable vibrational hot bands in the spectrum. A vibrational temperature of $500 \pm 50 \mathrm{~K}$ was estimated.

The ground state of $\mathrm{PbSe}$ is ${ }^{1} \Sigma^{+}$, described by the $1 \sigma^{2} 2 \sigma^{2} 3 \sigma^{2} 1 \pi^{4}$ configuration, where only the valence electrons are shown. The ionization of a $\pi$ electron gives rise to the spin-orbit split ${ }^{2} \Pi_{3 / 2}$ and ${ }^{2} \Pi_{1 / 2}$ bands. The removal of a $3 \sigma$ electron results in the ${ }^{2} \Sigma^{+}$state, which is a sharp band. The two $\Pi$ bands are broad and partially overlapped. From the spectrum, vibrational frequencies and accurate ionization potentials for the ionic states can be obtained. These are given in TABLE 1 for $\mathrm{PbSe}^{+}$.

\section{TABLE 1}

Spectroscopic constants of $\mathrm{PbSe}^{+}$.

\begin{tabular}{llll}
\hline & $\omega_{e}\left(\mathrm{~cm}^{-1}\right)$ & ${\text { IPad }(\mathrm{eV})^{\mathrm{a}}}^{2}$ & IPvt (eV) \\
\cline { 2 - 4 } & $210(6)$ & $8.671(7)$ & $8.800(7)$ \\
${ }^{\mathrm{b}} \Pi_{3 / 2}$ & $210(6)$ & $9.001(7)$ & $9.104(7)$ \\
$2 \Pi_{1 / 2}^{+}$ & $242(5)$ & $9.543(7)$ & \\
\hline
\end{tabular}

\footnotetext{
a adiabatic ionization potentials.

$b_{\text {vertical ionization potentials. }}$
}

We have done a series of the heavy Group IV-VI diatomics. The main interest was to investigate relativistics effect. Relativistic quantum chemical calculations have been performed for these molecules. The details of the 
calculations and the data analysis and comparisons between the calculated results and the experimental observations will be published later (10).

\subsection{As? and As 4}

The study of clusters is an emerging tield. One of the difficulties in doing photoelectron spectroscopy of neutral cluster species is the size-selectivity. The Group V elements offer a rare opportunity, where the vapor phase of these elements contains mainly single-sized species (11). For arsenic, the vapor phase is composed of only As4 clusters below the boiling temperature. As2 can be produced by pyrolysis of As4. As shown before (12), a cleaner source of As2 is from evaporating copper arsenide (Cu3As). Dyke et. al. (12), using this method, obtained the low resolution photoelectron spectra of As2 and As4.

Figs. 6 and 7 show the photoelectron spectra of As2 and As4 measured with our source. The As2 spectrum was taken at a temperature of about $1400 \mathrm{~K}$ by evaporating Cu3As (purchased from CERAC). The nozzle size with crucible configuration 2(a) was $0.13 \mathrm{~mm}$ and the carrier gas was 350 torr Ne. The As4 spectrum was taken at a temperature of about $650 \mathrm{~K}$, a nozzle size of $0.16 \mathrm{~mm}$ with crucible configuration 2(c), and a carrier gas of 200 torr He.

The As2 molecule is isoelectronic with $\mathrm{PbSe}$ and the valence electronic configuration can be written as $1 \sigma_{g}{ }^{2} 1 \sigma_{u}{ }^{2} 2 \sigma_{g}{ }^{2} 1 \pi_{u}{ }^{4}$. The removal of a $2 \sigma_{g}$ electron gives rise to the sharp ${ }^{2} \Sigma_{\mathrm{g}}{ }^{+}$band with little vibrational excitation. The $1 \pi_{\mathrm{u}}$ orbital is a bonding orbital and extensive vibrational structure was observed for the related spin-orbit split ${ }^{2} \prod_{u 3 / 2}$ and ${ }^{2} \prod_{u 1 / 2}$ bands. These two bands are heavily overlapped, which makes it hard to determine the adiabatic ionization potential of the ${ }^{2} \prod_{u 1 / 2}$ state. From the vibrational structure of the ${ }^{2} \Pi_{u 3 / 2}$ band, a vibrational frequency of $450 \mathrm{~cm}^{-1}$ was derived. This value is even bigger than that of the neutral ground state, which has a vibrational frequency of $429.55 \mathrm{~cm}^{-1}$ (13). This is in apparent contradiction with the fact that the $1 \pi_{\mathrm{u}}$ orbital is a bonding orbital. Accurate $a b$ initio calculation is needed to explain and confirm this observation. The ionization potentials for the ${ }^{2} \Pi_{\mathrm{u} 3 / 2}$ and ${ }^{2} \Sigma_{\mathrm{g}}{ }^{+}$states are $9.511(6)$ and $10.228(5) \mathrm{eV}$, respectively.

As4 is a tetrahedral molecule. In $T_{d}$ symmetry, the valence electron configuration of As4 can be represented as $\left(1 a_{1}\right)^{2}\left(1 t_{2}\right)^{6}\left(2 a_{1}\right)^{2}\left(2 t_{2}\right)^{6}(1 e)^{4}$, where the $\mathrm{la}_{1}$ and $1_{2} \mathrm{t}_{2}$ orbitals are mainly comprised of $A s(4 s)$ character and the $2 \mathrm{a}_{1}, 2 \mathrm{t}_{2}$ and le orbitals are mostly composed of As(4p) character. Only the ionizations of the later three were observed, as shown in Fig. 7. There are three vibrational modes for As4, $v_{1}\left(a_{1}\right)\left(340 \mathrm{~cm}^{-1}\right), v_{2}(e)\left(200 \mathrm{~cm}^{-1}\right)$, and $v_{3}\left(f_{2}\right)\left(250 \mathrm{~cm}^{-1}\right)(14)$. The ionization of the $2 a_{1}$ orbital results in a beautifully resolved band, which consists of a single $v_{1}$ vibrational progression. The derived vibrational frequency is $350 \pm 10$ $\mathrm{cm}^{-1}$, which is almost identical with that of the ground state. The $(1 \mathrm{e})^{-1}$ and $(2 \mathrm{t} 2)^{-1}$ bands are more complex because of the Jahn-Teller effect $(12,15)$. The spin-orbit 


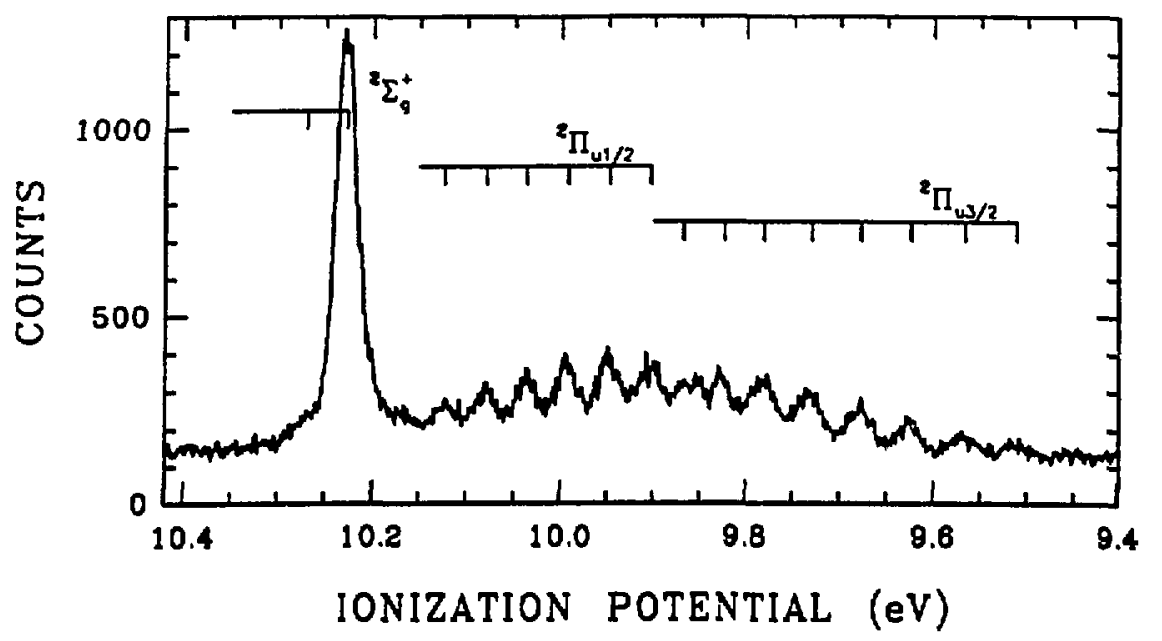

Fig. 6. The Hel photoelectron spectrum of As2.

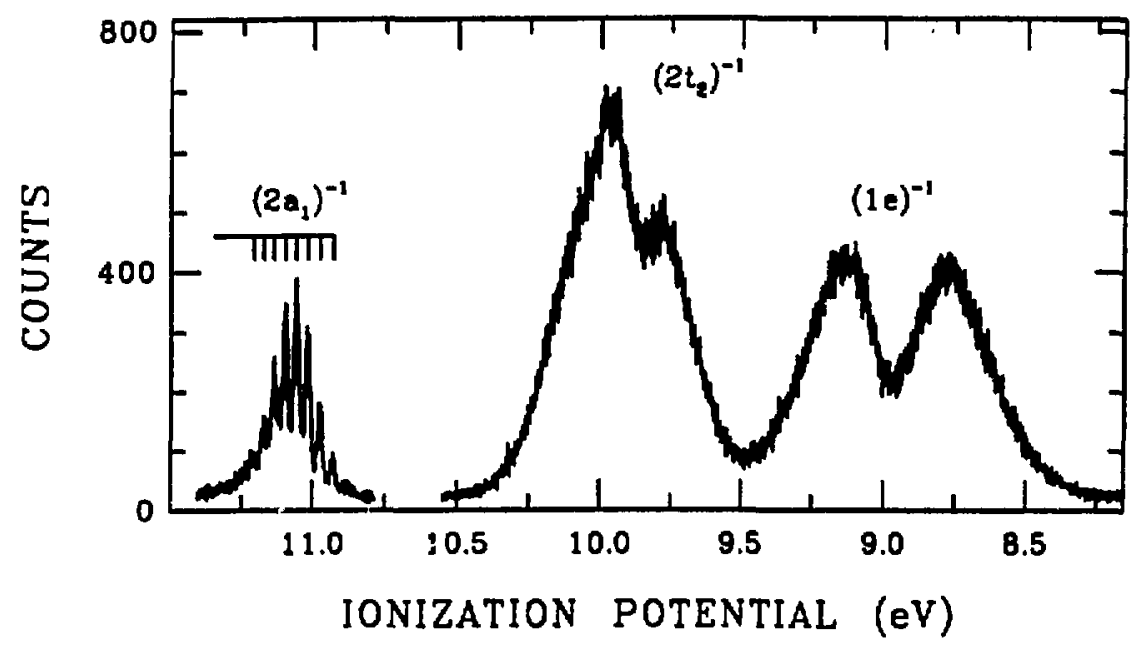

Fig. 7. The HeI Photoelectron spectrum of As4.

effect is also possible in the $\left(2 t_{2}\right)^{-1}$ band. Vibrational structure was partially resolved in these bands. The $\left(2 \mathrm{t}_{2}\right)^{-1}$ band was assigned to be due to the spin-orbit splitting along previously (12). A shoulder is visible at the high ionization side in the current spectrum. This suggests that the Jahn-Teller effect is also important. $\mathrm{As}_{4}$ is a prototype of tetrahedral $\mathrm{M}_{4}$ clusters. The Jahn-Teller effect should be important in all the degenerate states of this class of clusters. Tu understand the Jahn-Teller effect, it is essential to know the vibrational modes involved in the vibronic interaction. Fron the partially resolved vibrational structure for $\mathrm{As}_{4}{ }^{+}$, it 
is concluded that the $v_{2}$ mode is active in the Jahn-Teller interaction of the (le)-1 band, while the $v_{3}$ mode is involved in the $\left(2 t_{2}\right)^{-1}$ band. This is in agreement with the better-resolved spectrum of $\mathrm{P}_{4}^{+}(16)$, which is similar with $\mathrm{As}_{4}{ }^{+}$, but has larger vibrational frequencies.

\section{$3.3 \mathrm{ZnCl} 2$}

The Group IIB elements dihalides have been subjects of many previous PES investigations (17-24) to elucidate the ligand-field effect and the role of the $d^{10}$ shell in the chemical bonding. We examined $\mathrm{ZnCl}_{2}$ as part of our effort to study the first role transition metal dihalides, which are all expected to be more complicated than $\mathrm{ZnCl}_{2}$. We also wanted to see to what extent we could resolve the vibrational fine structure in the $\mathrm{ZnCl}_{2}$ photoelectron spectrum with our superior resolution.

$\mathrm{ZnCl}_{2}$ is a linear and symmetric molecule. Its valence electronic configuration can be written as $\mathrm{d}^{10} \sigma_{g}{ }^{2} \sigma_{u}{ }^{2} \pi_{u}{ }^{4} \pi_{g}{ }^{4}$. The $\sigma_{u}, \pi_{u}$ and $\pi_{g}$ orbitals are mainly composed of $\mathrm{Cl}(4 \mathrm{p})$ character and the $\sigma_{\mathrm{g}}$ orbital has both $\mathrm{Zn}(4 \mathrm{~s})$ and $\mathrm{Cl}(4 \mathrm{p})$ characters. All these orbitals are accessible with the Hel photon. The spectrum appears in Figs. 8. It was taken at $\sim 700 \mathrm{~K}$ with a crucible of type $2(\mathrm{c})$. The nozzle size was $0.17 \mathrm{~mm}$ and the carrier gas was 100 torr $\mathrm{He}$.

As can be seen from Fig. 8(a), vibrational structure was not fully resolved in these three bands. The spin-orbit effect is responsible in the ${ }^{2} \Pi_{g}$ and ${ }^{2} \Pi_{u}$ bands, which have very small spin-orbit splitting constants (23). The spin-orbit splitting is partially resolved in the ${ }^{2} \Pi_{\mathrm{g}}$ band. For the ${ }^{2} \Sigma_{\mathrm{u}^{+}}{ }^{+}$band, two factors should be responsible for its diffusiveness: (1) the vibrational frequencies become smaller in this final state than that of the ground state, (2) more than one vibrational mode gets excited during the photoionization process, especially the low frequency bending mode.

A well-resolved band was observed for the ${ }^{2} \Sigma_{\mathrm{g}}{ }^{+}$state, as can be seen from Fig. $8(\mathrm{~b})$. The resolved feature can not be assigned to a single vibrational progression on the basis of the spacings. The assignnent shown in Fig. 8(b) is according to the vibrational quanta of $\left(v_{1} v_{2} v_{3}\right)$. It should be remembered that only even quanta of the $v_{3}$ mode are allowed. We can see that the vertical transition is to the $(002)$ level, which suggests that the $\mathrm{ZnCl}_{2}{ }^{+}$is no longer symmetric in the ${ }^{2} \Sigma_{\mathrm{g}^{+}}$electronic state. From this assignment, we obtained the three vibrational frequencies: $v_{1}=380 \mathrm{~cm}^{-1}, v_{2}=280 \mathrm{~cm}^{-1}, v_{3}=530 \mathrm{~cm}^{-1}$, which are very close to those of the ground state (25).

As discussed in previous papers $(20,24)$, the $\mathrm{d}^{10}$ shell in $\mathrm{ZnCl}_{2}$ is split by the ligand-field into three levels: $\sigma_{g}, \pi_{u}$ and $\delta_{g}$. In $\mathrm{ZnCl}_{2}{ }^{+}$, the ${ }^{2} \prod_{\mathrm{u}}$ and ${ }^{2} \Delta_{\mathrm{g}}$ states can be further split by the spin-orbit effect. The combined ligand-field and spin-orbit effects would result in five bands in the photoelectron spectrum of the $d$ orbital. Bancroft et. al. first resolved all five bands (24). As can be seen in Fig. 8(c), these 


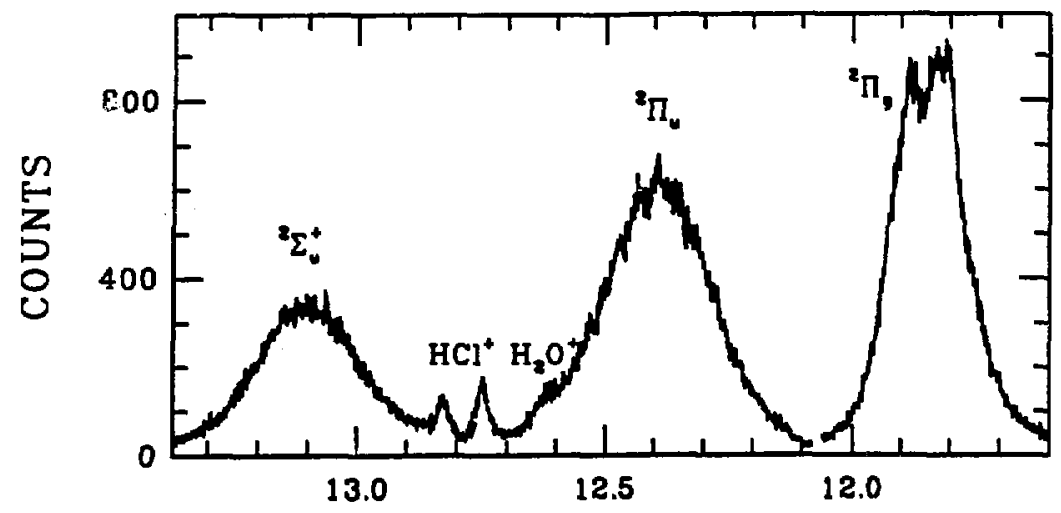

(a)

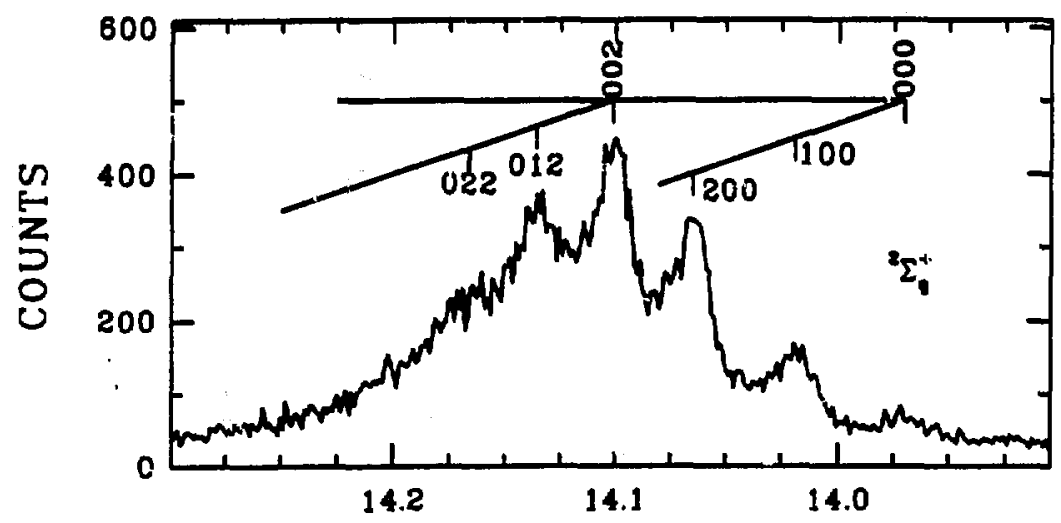

(b)

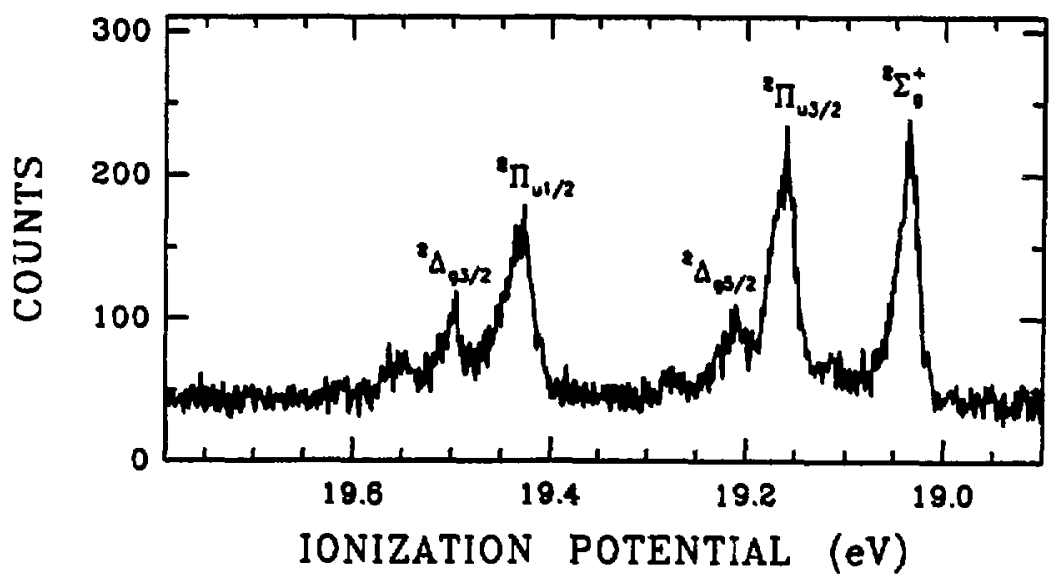

(c)

Fig. 8. The HeI photoelectron spectrum of $\mathrm{ZnCl}_{2}$. 
are very well-resolved in the current spectrum. In contrary to the assignment by Bancroft et. al., there do not seem to be any sizable vibrational excitations in any bands. This agrees with the fact that the $d$ orbitals are very inert in term of chemical bonding (20). An extra visible peak appears at $19.55 \mathrm{eV}$. This peak can not be assigned to any vibrational levels. A similar feature was also observed in $\mathrm{ZnI}_{2}{ }^{+}(20)$. It is likely to be due to a multielectron process.

\section{CONCLUSION}

We have described a new high temperature molecular beam source, which can produce an intense, continuous and moderately internally-cooled beams of high temperature species for high resolution UV photoelectron spectroscopic studies. The high resolution photoelectron spectra of $\mathrm{PbSe}, \mathrm{As}_{2}, \mathrm{As}_{4}$, and $\mathrm{ZnCl} 2$ were shown to demonstrate the performance of the source. In all the spectra discussed, vibrational fine structure was resolved, which was not possible before. This source can be used up to $2000 \mathrm{~K}$ or higher and provides us new opportunities for more detailed photoelectron spectroscopic studies of high temperature species and cluster systems.

\section{ACKNOWLEDGMENT}

This work was supported by the Director, Office of Energy Research, Office of Basic Energy Sciences, Chemical Sciences Division of the U.S. Department of Energy under Contract No. DE-AC03-76SF00098.

\section{REFERENCES}

1 See for example, a. J.M. Dyke, N. Jonathan, and A. Morris, Int. Rev. Phys. Chem., 2 (1982) 3-42;

b. G.M. Bancroft, D.J. Bristow, and L.L. Coatsworth, Chem. Phys. Lett., 82 (1981) 344;

c. J. Berkowitz, C.H. Batson, and G.L. Goodman, J. Chem. Phys., 71 (1979) 2624;

d. A.W. Potts and M.L. Lyus, J. Electron. Spectrosc. Relat. Phenom., 13 (1978) 30S;

e. S. Suzer, S.-T. Lee, and D.A. Shirley, J. Chem. Phys.,65 (1976) 412.

2 a. G.C. Allen, E.J. Baerends, P. Yernooijs, J.M. Dyke, A.M. Ellis, M. Feher, and A. Morris, J. Chem. Phys., 89 (1988) 5363;

b. A. Morris, J.M. Dyke, G.D. Josland, M.P. Hastings, and P.D. Francis, High Temp. Sci., 22 (1986) 95.

3 L.S. Wang, B. Niu, Y.T. Lee, and D.A. Shirley, Chem. Phys. Lett., 158 (1989) 297.

4 J.E. Pollard, D.J. Trevor, Y.T. Lee, and D.A. Shirley, Rev. Sci. Instrum., 52 (1981) 1837.

5 See for example, a. J.E. Pollard, D.J. Trevor, J.E. Reutt, Y.T. Lee, and D.A. Shirley, J. Chem. Phys., 77 (1982) 34.

b. J.E. Reutt, L.S. Wang, Y.T. Lee, and D.A. Shirley, J. Chem. Phys., 85 (1986) 6928. c. L.S. Wang, Y.T. Lee, and D.A. Shirley, J. Chem. Phys., 87 (1987) 2489.

6 J.B. Hopkins, P.R.R. Langridge-Smith, M.D. Morse, and R.E. Smalley, J. Chem. Phys. 78 (1983) 1627.

7 D.R. Preuss, S.A. Pace, and J.L. Gole. J. Chem. Phys., 71 (1979) 3553.

8 D. Bulgin, J. Dyke, F. Goodfellow, N. Jonathan, E. Lee, and A. Morris, J. Electron. Spectrosc. Relat. Phenom., 12 (1977) 67.

9 J.L. Margrave and R.H. Hauge, in: B. W. Rossiter (Ed.), Techniques of Chemistry, Vol. IX: Chemical Experimentation under Extreme Condition, John Wiley \& Sons, New York, 1980, pp. 300-301. 
L.S. Wang, B. Niu, Y.T Lee, D.A. Shirley, and K. Balasubramanian, in preparation. A.N. Nesmeyanov, R. Gary (Ed.), Vapor Pressure of the Chemical Elements, Elsevier, Amsterdam, 1963, pp. 286-306. J.M. Dyke, S. Elbel, A. Morris, and J.C.H. Stevens, J. Chem. Soc., Faraday Trans. 2, 82 (1986) 637.

13 K. P. Huber and G. Herzberg, Spectroscopic Constants of Diatomics, Van Nostrand Reinhold, New York, 1979.

14 V.E. Bondybey, G.P. Schwarzz, and J.E. Griffiths, J. Mol. Spectrosc., 89 (1981) 328. H.A. Jahn and E. Teller, Proc. Roy. Soc. A, 161 (1937) 220. L.S. Wang, B. Niu, Y.T. Lee, and D.A. Shirley, to be published.

17 G.W. Boggess, J.D. Allen Jr., and G.K. Schweitzer, J. Electron. Spectrosc. Relat. Phenom., 2 (1973) 467.

18 S. Evans and A.F. Orchard, J. Electron. Spectrosc. Relat. Phenom., 2 (1975) 207.

19 J. Berkowitz, J. Chem. Phys., 61 (1974) 407.

20 A.F. Orchard and N.V. Richardson, J. Electron. Spectrosc. Relat. Phenom., 6 (1975) 61.

21 J.M. Dyke, N.K. Fayad, G.D. Josland, and A. Morris, J. Chem. Soc. Faraday II, 76 (1980) 1672.

22 E.P.F. Lee and A.W. Potts, J. Electron. Spectrosc. Relat. Phenom., 22 (1981) 247.

23 D.J. Bristow, G.M. Bancroft, and J.S. Tse, Chem. Phys., 75 (1983) 263.

24 G.M. Bancroft, D.J. Bristow, and J.S. Tse, Chem. Phys., 75 (1983) 277.

25 G.E. Leroi, T.C. James, J.T. Hougen, and W. Klemperer, J. Chem. Phys., 36 (1962) 2879. 